05

\title{
Обменная пружина в гетероструктуре с низкотемпературным ферромагнетиком $\mathrm{Pd}_{0.96} \mathrm{Fe}_{0.04}$
}

\author{
(C) И.В. Янилкин, ${ }^{1}$ Э.Т. Мухаметова ${ }^{1}$, И.Р. Вахитов ${ }^{1}$, А.И. Гумаров ${ }^{1}$, \\ Р.В. Юсупов ${ }^{1}$, Л.Р. Тагиров ${ }^{1-3}$ \\ ${ }^{1}$ Институт фризики Казанского федерального университета, Казань, Россия \\ ${ }^{2}$ Казанский физико-технический институт ФИЦ КазНЦ РАН, Казань, Россия \\ ${ }^{3}$ Институт прикладных исследований АН Республики Татарстан, Казань, Россия \\ E-mail: yanilkin-igor@yandex.ru
}

Поступило в Редакцию 28 декабря 2018г.

В окончательной редакции 28 декабря 2018 г.

Принято к публикации 22 января 2019 г.

\begin{abstract}
Сообщается о получении обменного сдвига петли магнитного гистерезиса и эффекта магнитной пружины в тонкопленочных гетероструктурах, включающих слои низкотемпературного ферромагнетика $\mathrm{Pd}_{0.96} \mathrm{Fe}_{0.04}$, металлического кобальта и монооксида кобальта $(\mathrm{CoO})$ на подложке из монокристаллического сапфира. Образцы были выращены при последовательном применении методов магнетронного распыления и молекулярнолучевой эпитаксии на сверхвысоковакуумной установке. Установлено, что обменная анизотропия для слоя $\mathrm{Pd}_{0.96} \mathrm{Fe}_{0.04}$ в тонкопленочной гетероструктуре $\mathrm{Pd}_{0.96} \mathrm{Fe}_{0.04} / \mathrm{CoO} / \mathrm{Co}_{2} \mathrm{Al}_{2} \mathrm{O}_{3}$ отсутствует, и обменная пружина в такой системе не реализуется. На примере структуры $\mathrm{Pd}_{0.96} \mathrm{Fe}_{0.04} / \mathrm{Co} / \mathrm{CoO} / \mathrm{Al}_{2} \mathrm{O}_{3}$ показано, что по результатам магнитометрических измерений инверсия антиферромагнитного и ферромагнитного слоев приводит к закреплению магнитного момента слоя $\mathrm{Pd}_{0.96} \mathrm{Fe}_{0.04}$ на интерфейсе со слоем кобальта и реализации обменной пружины. Дополнительные аргументы в пользу такой интерпретации получены с применением микромагнитного моделирования петель магнитного гистерезиса.
\end{abstract}

DOI: 10.21883/PJTF.2019.08.47613.17664

Сплав $\operatorname{Pd}_{1-x}$ Fe при $x<0.1$ является магнитомягким низкотемпературным ферромагнетиком (ФМ) [1] и перспективен для использования в элементах сверхпроводящей спинтроники и быстрой одноквантовой логики (RSFQ - rapid single-flux quantum) [2-7]. Изменяя концентрацию железа в сплаве и условия его приготовления, можно контролировать такие ключевые параметры, как температура Кюри $T_{C}$ и намагниченность насыщения $M_{s}[8,9]$, а также варьировать величину коэрцитивного поля и магнитокристаллической анизотропии [1]. Важной составляющей для реализации спинтронных устройств на базе тонкопленочных гетероструктур является возможность закрепления заданного направления намагниченности ФМ-слоя, что обычно достигается с использованием эффекта обменного сдвига (exchange bias) на границе ФМ- и антиферромагнитного слоев [10,11]. Наиболее изученным и удобным антиферромагнетиком (АФМ) для таких целей является монооксид кобальта (CoO - антиферромагнитный изолятор) благодаря температуре Нееля $\left(T_{N}=291 \mathrm{~K}\right)$, близкой к комнатной, и возможности создания большого по величине обменного сдвига (см. обзор [11] и ссылки в нем). В классическом варианте обменный сдвиг реализуется при условии $T_{C}(\Phi \mathrm{M})>T_{N}(\mathrm{~A} \Phi \mathrm{M})$ в результате охлаждения гетероструктуры ФМ/АФМ в магнитном поле от $T>T_{N}$ до $T<T_{N}$. Однако для сплава $\mathrm{Pd}_{1-x} \mathrm{Fe}_{x}$ с $0.01<x<0.08 T_{C}=18-200 \mathrm{~K}[1]$, т.е. реализуется обратное соотношение: $T_{C}(\Phi \mathrm{M})<T_{N}(\mathrm{AФМ)}$, поэтому создание обменного сдвига требует более сложной структуры [12] со вспомогательным слоем „высокотемпературного“ ферромагнетика с $T_{C}(\Phi \mathrm{M} 2)>T_{N}(\mathrm{~A} \Phi \mathrm{M})$, призванного создать нескомпенсированный магнитный момент в находящемся с ним в контакте антиферромагнетике при прохождении его температуры Нееля $T_{N}(\mathrm{AФМ)} \mathrm{в} \mathrm{процессе} \mathrm{замораживания} \mathrm{системы.} \mathrm{Действи-}$ тельно, направление намагниченности сплава $\mathrm{Cu}_{41} \mathrm{Ni}_{59}$ с низкой температурой Кюри $\left(T_{C}=110-120 \mathrm{~K}\right)$ удалось „закрепить“ с помощью бислоя $\mathrm{Co} / \mathrm{CoO}$ в гетероструктуре $\mathrm{Co} / \mathrm{CoO}_{x} / \mathrm{Cu}_{41} \mathrm{Ni}_{59}$ [13]. В настоящей работе показано, что для сплава $\mathrm{Pd}_{1-x} \mathrm{Fe}_{x}$ такой прием не приводит к успеху. Однако обменного закрепления магнитного момента сплава $\mathrm{Pd}_{1-x} \mathrm{Fe}_{x}$ удается достичь, изменив порядок следования слоев в гетероструктуре с $\mathrm{Pd}_{0.96} \mathrm{Fe}_{0.04}\left(t_{F}\right) / \mathrm{CoO} / \mathrm{Co} / \mathrm{Al}_{2} \mathrm{O}_{3}$ на $\mathrm{Pd}_{0.96} \mathrm{Fe}_{0.04}\left(t_{F}\right) / \mathrm{Co} / \mathrm{CoO} / \mathrm{Al}_{2} \mathrm{O}_{3}$, где $t_{F}$ - толщина слоя сплава $\mathrm{Pd}_{0.96} \mathrm{Fe}_{0.04}$. В такой конструкции магнитомягкий слой сплава $\mathrm{Pd}_{1-x} \mathrm{Fe}_{x}$ находится в непосредственном контакте с обменно-закрепленным слоем кобальта, т.е. реализуется ситуация бислоя мягкий-жесткий ферромагнетик (soft-hard bilayer), которая приводит к эффекту обменной пружины [14]. Последняя улучшает характеристики ячеек магнитной памяти, основанных на переносе вращательного момента [15]. Интерпретация результатов магнитометрических измерений в терминах обменной пружины подтверждается микромагнитными расчетами петель магнитного гистерезиса.

Многослойные магнитные гетероструктуры были изготовлены на сверхвысоковакуумной установке 

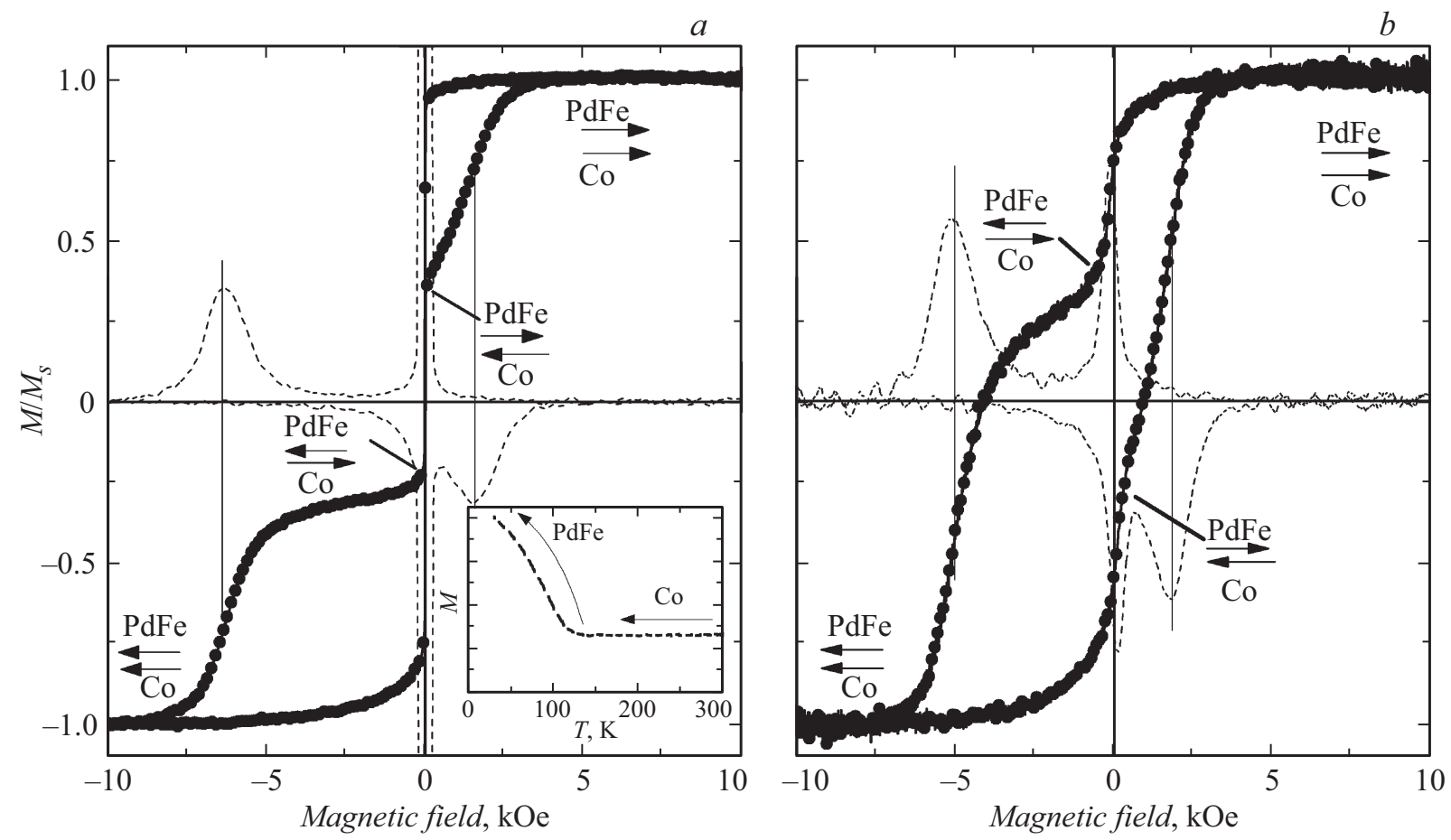

Рис. 1. Петли магнитного гистерезиса, измеренные при $30 \mathrm{~K}$ для образцов $\mathrm{Pd}_{0.96} \mathrm{Fe}_{0.04} / \mathrm{CoO} / \mathrm{Co} / \mathrm{Al}_{2} \mathrm{O}_{3}(a) \quad$ и $\mathrm{Pd}_{0.96} \mathrm{Fe}_{0.04} / \mathrm{Pd}_{0.85} \mathrm{Fe}_{0.15} / \mathrm{CoO} / \mathrm{Co} / \mathrm{Al}_{2} \mathrm{O}_{3}(b)$. Тонкими штриховыми линиями обозначены производные от кривых намагничивания (в отн. ед.) На вставке - зависимость магнитного момента гетероструктуры $\mathrm{Pd}_{0.96} \mathrm{Fe}_{0.04} / \mathrm{CoO} / \mathrm{Co} / \mathrm{Al}_{2} \mathrm{O}_{3}$ от температуры.

(SPECS/BESTEC, Германия) с использованием магнетронного распыления металлического кобальта в атмосфере аргона (чистота 99.9999\%) для осаждения ФМ-слоя Со либо в смеси $\mathrm{Ar}$ и $\mathrm{O}_{2}$ в соотношении 50:1 для АФМ-слоя $\mathrm{CoO}$. Осаждение слоя $\mathrm{Pd}_{1-x} \mathrm{Fe}_{x}$ осуществлялось соиспарением высокочистых металлов $\mathrm{Pd}(3 \mathrm{~N} 5)$ и $\mathrm{Fe}$ (3N8) из термоэффузионных ячеек в камере молекулярно-лучевой эпитаксии с остаточным давлением $\sim 5 \cdot 10^{-10} \mathrm{mbar}$. Содержание железа в сплаве задавалось подбором температур ячеек $\left(T_{\mathrm{Fe}}=1184^{\circ} \mathrm{C}\right.$, $\left.T_{\mathrm{Pd}}=1320^{\circ} \mathrm{C}\right)$. Стехиометрия оксида кобальта и соотношение элементов в сплаве контролировались с помощью рентгеновской фотоэлектронной спектроскопии, осуществлявшейся in situ в аналитической камере вакуумной установки. В качестве подложек использовались монокристаллические пластинки сапфира $\mathrm{Al}_{2} \mathrm{O}_{3}$ с ориентацией (0001) (epi-ready от Crystal, Германия). На всех этапах осаждения слоев подложка находилась при комнатной температуре. Исследования магнитных свойств образцов проводились на вибрационном магнитометре системы измерения физических свойств PPMS-9 компании Quantum Design в диапазоне температур 30-300 К после их охлаждения в магнитном поле $1 \mathrm{kOe}$, приложенном в плоскости пленки (field cooled). Суммарная толщина пленок контролировалась стилусным нанопрофилометром BRUKER Dektak XT (диаметр закругления стилуса $2 \mu \mathrm{m}$, точность измерения вертикальных смещений лучше $0.5 \mathrm{~nm}$ ).
Первый образец, $\quad \mathrm{Pd}_{0.96} \mathrm{Fe}_{0.04}(40 \mathrm{~nm}) / \mathrm{CoO}(3.3 \mathrm{~nm}) /$ $\mathrm{Co}(3 \mathrm{~nm}) / \mathrm{Al}_{2} \mathrm{O}_{3}$, был синтезирован нами по аналогии с работой [13], в которой высокотемпературный (Со) и низкотемпературный $\left(\mathrm{Cu}_{41} \mathrm{Ni}_{59}\right)$ ферромагнетики были нанесены по разные стороны от АФМ-слоя $\mathrm{CoO}$ и наблюдался обменный сдвиг (разной величины) для обоих ферромагнетиков. Однако в нашем образце со слоем сплава $\mathrm{Pd}_{0.96} \mathrm{Fe}_{0.04}$ в качестве магнитомягкого слоя обменный сдвиг петли магнитного гистерезиса отсутствует (рис. 1,a). До конца причина отсутствия обменного сдвига нам неясна. На вставке к рис. 1, $a$ показана типичная для всех полученных в настоящей работе образцов температурная зависимость $M(T)$, содержащая постоянный, практически не зависящий от температуры в данном диапазоне вклад от кобальта и низкотемпературный сигнал от сплава $\mathrm{Pd}_{0.96} \mathrm{Fe}_{0.04}$. Температура Кюри слоя $\mathrm{Pd}_{0.96} \mathrm{Fe}_{0.04}$ составила $124 \mathrm{~K}$ (рис. $1, a$, вставка), что является характерной величиной для данной концентрации железа в сплаве $[1,8]$.

Для реализации обменной связи антиферромагнитного $\mathrm{CoO}$ с $\mathrm{Pd}_{1-x} \mathrm{Fe}_{x}$ был добавлен дополнительный промежуточный слой $\mathrm{Pd}_{0.85} \mathrm{Fe}_{0.15}$ с относительно высокой концентрацией железа, который является ферромагнитным уже при комнатной температуре. Кроме того, чтобы увеличить величину обменного сдвига, слой $\mathrm{Pd}_{0.96} \mathrm{Fe}_{0.04}$ был сделан более тонким. В результате получилась следующая структура: $\mathrm{Pd}_{0.96} \mathrm{Fe}_{0.04}(10 \mathrm{~nm}) / \mathrm{Pd}_{0.85} \mathrm{Fe}_{0.15}(2 \mathrm{~nm}) /$ $\mathrm{CoO}(3.3 \mathrm{~nm}) / \mathrm{Co}(3 \mathrm{~nm}) / \mathrm{Al}_{2} \mathrm{O}_{3}$. Экспериментальная петля магнитного гистерезиса этой структуры приведена на 

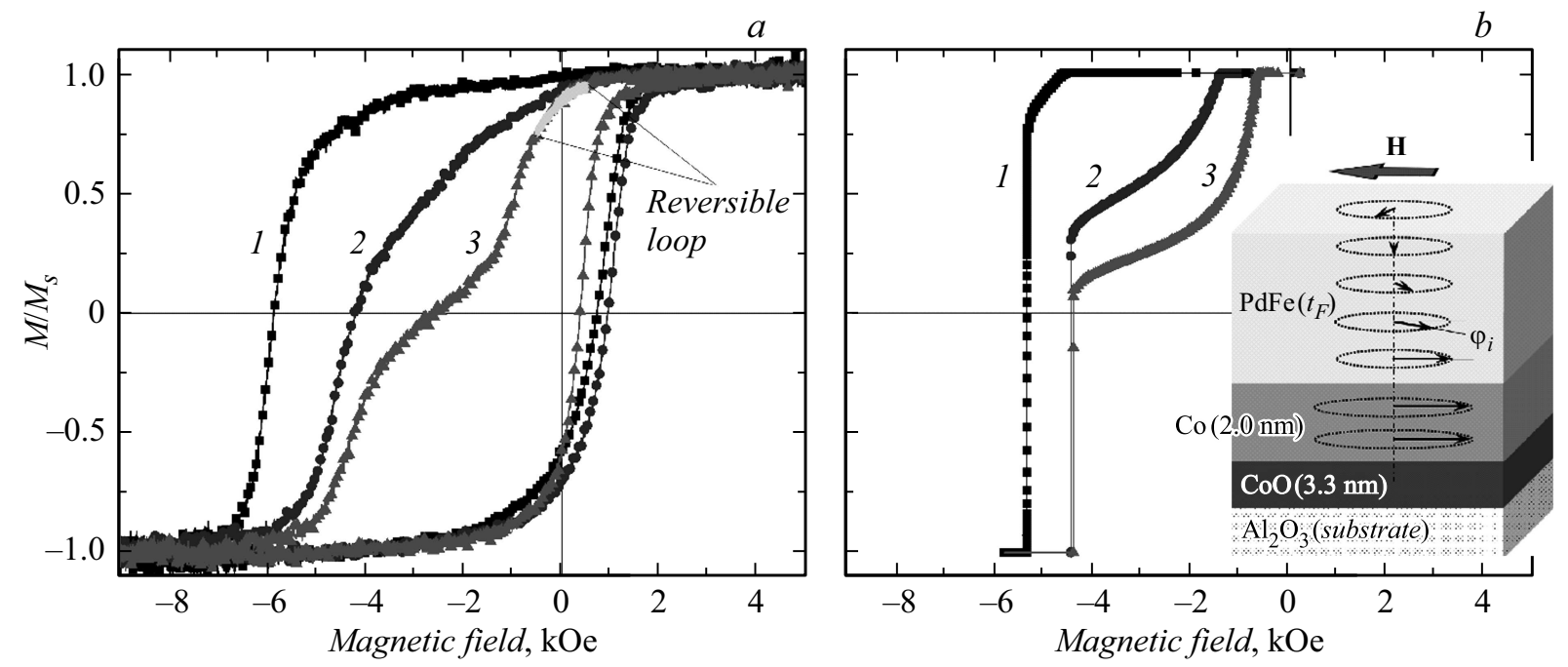

Рис. 2. Петли магнитного гистерезиса, измеренные при температуре $30 \mathrm{~K}$, для образцов $\mathrm{Pd}_{0.96} / \mathrm{Fe}_{0.04} / \mathrm{Co} / \mathrm{CoO} / \mathrm{Al}_{2} \mathrm{O}_{3}$ с толщинами $t_{F}$ слоя $\mathrm{Pd}_{0.96} \mathrm{Fe}_{0.04}$, равными $5(1), 10(2)$ и $15 \mathrm{~nm}(3)(a)$, и их микромагнитное моделирование $(b)$. На вставке - иллюстрация модели обменной пружины.

pис. $1, b$. По сравнению с полученной для предыдущего образца форма петли от пленки $\mathrm{Pd}_{1-x} \mathrm{Fe}_{x}$ немного изменилась, и переворот момента происходит не так резко. Это указывает на возникновение слабого взаимодействия с АФМ-слоем, однако величина обменного смещения для $\mathrm{Pd}_{1-x} \mathrm{Fe}_{x}$ чрезвычайно мала.

Чтобы обеспечить обменный сдвиг петли гистерезиса сплава, мы ввели прослойку ферромагнитного Со между $\mathrm{Pd}_{0.96} \mathrm{Fe}_{0.04}$ и антиферромагнитным $\mathrm{CoO}$ (рис. 2, а) в гетероструктурах $\mathrm{Pd}_{0.96} \mathrm{Fe}_{0.04}(5,10,15 \mathrm{~nm}$ )/ $\mathrm{Co}(2 \mathrm{~nm}) / \mathrm{CoO}(3.3 \mathrm{~nm}) / \mathrm{Al}_{2} \mathrm{O}_{3}$. Величина обменного сдвига в данном случае сильно зависит от толщины слоя $\mathrm{Pd}_{0.96} \mathrm{Fe}_{0.04}$. Форма петель магнитного гистерезиса указывает на существование в гетероструктуре магнитной пружины типа мягкий $\left(\mathrm{Pd}_{1-x} \mathrm{Fe}_{x}\right)$ /жесткий (Co) ферромагнетик [14]. Характерной особенностью магнитной пружины является то, что в результате обменного взаимодействия магнитный момент мягкого магнитного слоя закрепляется на границе с магнитожестким. В свою очередь магнитные моменты мягкого ферромагнетика в более удаленных от границы раздела слоях могут вращаться на некоторый угол $\left(\varphi_{i}\right)$ под действием приложенного внешнего поля (рис. $2, b$, вставка). Такое поведение системы в силу обратимости вращения моментов называется обменной пружиной. На рис. $2, a$ в качестве примера показана обратимость петли гистерезиса для структуры $\mathrm{Pd}_{0.96} \mathrm{Fe}_{0.04}(15 \mathrm{~nm}) / \mathrm{Co} / \mathrm{CoO} / \mathrm{Al}_{2} \mathrm{O}_{3}$ в диапазоне полей от -500 до $500 \mathrm{Oe}$ (серые точки).

Если представить гетероструктуру $\mathrm{Pd}_{0.96} \mathrm{Fe}_{0.04} / \mathrm{Co} /$ $\mathrm{CoO} / \mathrm{Al}_{2} \mathrm{O}_{3}$ в виде подслоев с вращающимися спинами (рис. 2, $b$, вставка), то полную энергию системы можно записать как сумму обменных энергий интерфейсов внутренних подслоев $\mathrm{Pd}_{0.96} \mathrm{Fe}_{0.04}$ и $\mathrm{Co}$, a также энергии дипольного взаимодействия подслоев с внешним полем $H$

$$
\begin{aligned}
E= & -\sum_{i=1}^{N_{1}+N_{2}} M_{i} H \cos \left(\varphi_{i}-\varphi_{H}\right) \\
& -1 / d^{2}\left\{\sum_{i=1}^{N_{1}} A_{\mathrm{Co}} \cos \left(\varphi_{\mathrm{Co}_{i}}-\varphi_{\mathrm{Co}_{i+1}}\right)\right. \\
& -\sum_{i=1}^{N_{2}} A_{\mathrm{PdFe}} \cos \left(\varphi_{\mathrm{PdFe}_{i}}-\varphi_{\mathrm{PdFe}_{i+1}}\right) \\
& -A_{\mathrm{CoO}-\mathrm{Co}} \cos \left(\varphi_{\mathrm{CoO}-\varphi_{\mathrm{Co}}}\right) \\
& \left.-A_{\mathrm{Co}-\mathrm{PdFe}} \cos \left(\varphi_{\mathrm{Co}}-\varphi_{\mathrm{PdFe}}\right)\right\},
\end{aligned}
$$

где $M_{i}-$ намагниченность подслоя, $A_{\mathrm{Co}}, A_{\mathrm{PdFe}}, A_{\mathrm{CoO}-\mathrm{Co}}$, $A_{\mathrm{Co}-\mathrm{PdFe}}-$ обменные константы между подслоями $\mathrm{Co}$, $\mathrm{PdFe}$ и на интерфейсах $\mathrm{CoO}-\mathrm{Co}$ и $\mathrm{Co}-\mathrm{PdFe}, d-$ толщина подслоя, а угол $\varphi_{i}$ отображает ориентацию намагниченности подслоя; $N_{1}$ и $N_{2}-$ количество подслоев, на которые разбиты слои Со и $\mathrm{PdFe}$ соответственно. Известно, что для кобальта $A_{\mathrm{Co}}=31 \mathrm{pJ} / \mathrm{m}$, $M_{\mathrm{Co}}=1400 \mathrm{kA} / \mathrm{m}[16]$. Тогда, задав измеренное значение $M_{\mathrm{PdFe}}=130 \mathrm{kA} / \mathrm{m}$ и подобрав значения обменной энергии $A_{\mathrm{Co}-\mathrm{PdFe}}=1 \mathrm{pJ} / \mathrm{m}, A_{\mathrm{PdFe}}=0.4 \mathrm{pJ} / \mathrm{m}$, мы промоделировали поведение магнитной системы при изменении поля от $200 \mathrm{Oe}$ до $-6 \mathrm{kOe}$ (рис. $2, b$ ). Моделирование выполнялось с помощью программного пакета OOMMF [17]. Площадь гетероструктуры равнялась $4 \times 4 \mu \mathrm{m}$, размер расчетной ячейки $2000 \times 2000 \times 0.5 \mathrm{~nm}$. Закрепление кобальта антиферромагнетиком задавалось с помощью создания одноосной анизотропии в близлежащем к АФМ подслое кобальта с константой анизотропии $K=1.9 \mathrm{MJ} / \mathrm{m}^{3}$. Формы модельных петель магнитного 
гистерезиса качественно воспроизводят основные черты экспериментальных (рис. 2, $a, b)$.

Таким образом, в гетероструктуре $\mathrm{Pd}_{0.96} \mathrm{Fe}_{0.04} / \mathrm{Co} / \mathrm{CoO}$ было обнаружено явление обменного сдвига у низкотемпературного ферромагнетика. При этом для толщин $\mathrm{Pd}_{0.96} \mathrm{Fe}_{0.04}$ более $\sim 10 \mathrm{~nm}$ система $\mathrm{Pd}_{0.96} \mathrm{Fe}_{0.04} / \mathrm{Co} / \mathrm{CoO}$ ведет себя как обменная пружина, что подтверждается данными микромагнитного моделирования.

Работа выполнена при финансовой поддержке РФФИ (проект № 18-32-01041 мол_а) с использованием оборудования ФЦКП ФХИ КФУ. И.Р. Вахитов и А.И. Гумаров благодарят Программу повышения конкурентоспособности КФУ за частичную поддержку исследований, Л.Р. Тагиров благодарит Программу Президиума РАН „Актуальные проблемы физики низких температур“ за частичную поддержку. Работа Р.В. Юсупова осуществлялась за счет средств субсидии, выделенной Казанскому федеральному университету для выполнения государственного задания в сфере научной деятельности (задание 3.7704.2017/4.6).

\section{Список литературы}

[1] Esmaeili A., Yanilkin I.V., Gumarov A.I., Vakhitov I.R., Gabbasov B.F., Yusupov R.V., Tagirov L.R. // J. Alloys Compd. In press.

[2] Larkin T.I., Bol'ginov V.V., Stolyarov V.S., Ryazanov V.V., Vernik I.V., Tolpygo S.K., Mukhanov O.A. // Appl. Phys. Lett. 2012. V. 100. N 22. P. 222601.

[3] Ryazanov V.V., Bol'ginov V.V., Sobanin D.S., Vernik I.V., Tolpygo S.K., Kadin A.M., Mukhanov O.A. // Phys. Procedia. 2012. V. 36. P. 35-41.

[4] Vernik I.V., Bol'ginov V.V., Bakurskiy S.V., Golubov A.A., Kupriyanov M.Yu., Ryazanov V.V., Mukhanov O.A. // IEEE Trans. Appl. Supercond. 2013. V. 23. N 3. Pt 1. P. 1701208.

[5] Niedzielski B.M., Diesch S.G., Gingrich E.C., Wang Y., Loloee R., Pratt W.P., Jr., Birge N.O. // IEEE Trans. Appl. Supercond. 2014. V. 24. N 4. P. 1800307.

[6] Golovchanskiy I.A., Bol'ginov V.V., Abramov N.N., Stolyarov V.S., Ben Hamida A., Chichkov V.I., Roditchev D., Ryazanov V.V. // J. Appl. Phys. 2016. V. 120. N 16. P. 163902.

[7] Glick J.A., Loloee R., Pratt W.P., Birge N.O. // IEEE Trans. Appl. Supercond. 2017. V. 27. N 4. Pt. 1. P. 1800205 (1-5).

[8] Ewerlin M., Pfau B., Güther C.M., Schaffert S., Eisebitt S., Abrudan R., Zabel H. // J. Phys.: Condens. Matter. 2013. V. 25. N 26. P. 266001.

[9] Esmaeili A., Yanilkin I.V., Gumarov A.I., Vakhitov I.R., Gabbasov B.F., Kïamov A.G., Rogov A.M., Osin Yu.N., Denisov A.E., Yusupov R.V., Tagirov L.R. // Thin Solid Films. 2019. V. 669. P. 338-344.

[10] Nogues J., Schuller I.K. // J. Magn. Magn. Mater. 1999. V. 192. N 2. P. 203-232.

[11] Radu F., Zabel H. // Magnetic heterostructures. Springer tracts in modern physics. V. 227 / Eds H. Zabel, S.D. Bader. Berlin: Springer, 2008. Ch. 3.

[12] Blamire M.G., Ali M., Leung C.-W., Marrows C.H., Hickey B.J. // Phys. Rev. Lett. 2007. V. 98. N 21. P. 217202.

[13] Zdravkov V.I., Lenk D., Morari R., Ullrich A., Obermeier G., Mïler C., Krug von Nidda H.A., Sidorenko A.S., Horn S., Tidecks R., Tagirov L.R. // Appl. Phys. Lett. 2013. V. 103. N 6. P. 062604.
[14] Fullerton E.E., Jiang J.S., Bader S.D. // J. Magn. Magn. Mater. 1999. V. 200. N 1-3. P. 392-404.

[15] Wang J.P. // Nature Mater. 2005. V. 4. N 3. P. 191-192.

[16] Coey J.M.D. Magnetism and magnetic materials. Cambridge University Press, 2010. $617 \mathrm{p}$.

[17] Donahue M.J. OOMMF user's guide. Version 1.0. 1999. N 6376. https://doi.org/10.6028/NIST.IR.6376 